\title{
Claudin-5 Affects Endothelial Autophagy in Response to Early Hypoxia
}

\author{
Ping Yu+, Yanyu Li+, Gaoliang Zhong, Wen Li, Bing Chen and Jingjing Zhang*
}

Affiliated Hospital of Guangdong Medical University \& Key Laboratory of Zebrafish Model for Development and Disease of Guangdong Medical University, Zhanjiang, China

Hypoxic injury to cerebrovascular endothelial cells (ECs) after stroke leads to blood-brain barrier (BBB) dysfunction, which is commonly associated with disruptions of endothelial tight junctions (TJs) and increased permeability. Therefore, maintaining the structural integrity and proper function of the BBB is essential for the homeostasis and physiological function of the central nervous system (CNS). Our previous study revealed that autophagy

OPEN ACCESS

Edited by:

Jincai Luo,

Peking University, China

Reviewed by: Abraham Jacob Al-Ahmad, Texas Tech University Health Sciences Center, United States Xinchun Jin,

Soochow University, China

*Correspondence: Jingjing Zhang

jingjing.zhang@live.com orcid.org/0000-0002-8789-4638

tThese authors have contributed equally to this work

Specialty section: This article was submitted to Vascular Physiology, a section of the journal

Frontiers in Physiology

Received: 07 July 2021 Accepted: 03 August 2021 Published: 31 August 2021

Citation:

Yu P, Li Y, Zhong G, Li W, Chen B and

Zhang J (2021) Claudin-5 Affects Endothelial Autophagy in Response to Early Hypoxia.

Front. Physiol. 12:737474. doi: 10.3389/fphys.2021.737474 functions on protecting the BBB by regulating the dynamics of Claudin-5, the essential TJ protein, under short-term starvation or hypoxia conditions. Here, we show that in zebrafish and in vitro cells, loss of membranous Claudin-5 conversely determine the occurrence of hypoxia-induced autophagy in cerebrovascular ECs. Absence of endothelial Claudin- 5 could partly attenuate endothelial cell apoptosis caused by short-term hypoxic injury. Mechanism studies revealed that under hypoxic conditions, the existence of membranous Claudin- 5 affects the stimulation of hypoxia inducible factor 1 subunit alpha (HIF-1a) and the inducible nitric oxide synthase (iNOS), which are responsible for the translocation of and endocytosis of caveole-packaged Claudin-5 into cytosol. Meanwhile, loss of Claudin-5 affects the generation of reactive oxygen species (ROS) and the downstream expression of BCL2/adenovirus E1B $19 \mathrm{kDa}$ protein interacting protein 3 (Bnip3). These together suppress the endothelial autophagy under hypoxia. This finding provides a theoretical basis for clarifying the mechanism of hypoxia-induced BBB injury and its potential protection mechanisms.

Keywords: Claudin-5, blood-brain barrier, autophagy, hypoxia, permeability

\section{INTRODUCTION}

As a main part of the neurovascular units, the blood-brain barrier (BBB) is a physical barrier for the central nervous system (CNS) and is mainly composed of endothelial cells (ECs), tight junction (TJ) proteins, capillary basement membranes, pericytes, and astrocytes (Sweeney et al., 2019; Lochhead et al., 2020). The BBB exchanges nutrients and ions and tightly restricts toxic substances, inflammatory factors and immune cells in the peripheral blood from entering the CNS to maintain homeostasis. TJs are mainly composed of the proteins Claudin, Occludin, and Zonula Occludens-1 (ZO-1), which form the TJ structural skeleton that limits and regulates the permeability between cells and maintains barrier function (Wilhelm et al., 2016; Kadry et al., 2020). In the BBB, Claudin-5 is an important structural protein in the TJs connecting 
microvascular ECs, and it influences the development of cerebral blood vessels and the function of the BBB (Greene et al., 2019; Haruwaka et al., 2019; Hashimoto and Campbell, 2020). The clinicopathological characteristics of the BBB after ischemic stroke are evacuation of the TJ structure between ECs, a decrease in Claudin-5 protein expression, and an increase in permeability (Lv et al., 2018; Gholami et al., 2020). Therefore, to explore the mechanism by which Claudin-5 participates in the damage of $\mathrm{BBB}$ during cerebral ischemia has important clinical importance for the diagnosis and treatment of acute stroke, thrombolytic reperfusion, prognosis, and the prevention and treatment of hemorrhagic transformation after cerebral infarction.

Autophagy is a process by which cytoplasmic proteins or organelles are engulfed in vesicles, which fuse with lysosomes to form autophagic lysosomes; the contents of the lysosomes are degraded, thereby fulfilling the metabolic needs of the cell itself and the renewal of certain organelles. An increasing number of studies have shown that autophagy plays an important role in cerebral vascular pathology after stroke (Feng et al., 2017; Kim et al., 2018; Wang et al., 2018; Shi et al., 2021). Our studies used in vitro hypoxia or serum starvation models to mimic the onset of ischemic stroke, and we showed that early hypoxia/starvation could induce autophagy and the redistribution of membranous caveolin-1 (Cav-1) and Claudin-5 into cytosol, and autophagosomes mediate the degradation of cytosolically accumulated Claudin-5, thereby eliminating reactive oxygen species (ROS) and protecting the functional integrity of the endothelial barrier (Yang et al., 2019b, 2020).

In our subsequent study, we found that loss of membranous Claudin-5 in cerebrovascular endothelial cells could conversely inhibit the occurrence of autophagy in response to hypoxia. However, the molecular mechanism by which Claudin- 5 affects endothelial autophagy is unclear. Here, by constructing both brain microvascular endothelial (bEnd.3) cell lines with stable Claudin-5 knockout and zebrafish model with knockdown of claudin-5b by specific morpholino (MO), we revealed an underlying molecular mechanism by which Claudin-5 affects endothelial autophagy during the early period of hypoxic injury.

\section{MATERIALS AND METHODS}

\section{Cell Culture and Treatment}

Mouse bEnd. 3 were cultured in Dulbecco's modified Eagle's medium (DMEM, Gibco, C11995500BT) supplemented with $10 \%$ fetal bovine serum (FBS, Gibco) at $37^{\circ} \mathrm{C}$ in $5 \% \mathrm{CO}_{2}$. The Claudin- $5^{112 \Delta 5}$-mutated bEnd. 3 cell line was generated by the

\footnotetext{
Abbreviations: BBB, Blood-brain barrier; CNS, Central nervous system; bEnd.3 cells, Brain microvascular endothelial cells; HIF-1a, Hypoxia inducible factor 1 subunit alpha; iNOS, Inducible nitric oxide synthase; Cav-1, Caveolin-1; ROS, Reactive oxygen species; ECs, Endothelial cells; TJ, Tight junction; ZO-1, Zonula occludens-1; MO, Morpholino; CRISPR/Cas9, Clustered regularly interspaced short palindromic repeats/CRISPR-associated protein 9; DCFH-DA, 2',7'-dichlorofluorescein diacetate; DMEM, Dulbecco's modified Eagle's medium; FBS, Fetal bovine serum; Bnip3, BCL2/adenovirus E1B $19 \mathrm{kDa}$ protein interacting protein 3; MFI, Mean fluorescence intensity; TEER, Trans-endothelial electrical resistance.
}

clustered regularly interspaced short palindromic repeats/CRISPRassociated protein 9 (CRISPR/Cas9) method using sgRNA (ccaCAACATCGTGACGGCGCAGA; Yang et al., 2021), and the Claudin- $5^{297 \Delta 4}$-mutated bEnd.3 cell line was generated by the CRISPR/Cas9 method using sgRNA (cctTGACCGGCGCTC $A G T G C A C C)$. The cells were stimulated with hypoxia as previously reported (Yang et al., 2019b, 2020). In brief, the cells were treated with $1 \% \mathrm{O}_{2} / 94 \% \mathrm{~N}_{2} / 5 \% \mathrm{CO}_{2}$. Then, the cells were incubated at $37^{\circ} \mathrm{C}$ in a constant temperature incubator for 2, 4, or $6 \mathrm{~h}$. Cell viability was examined by Cell Counting Kit-8 (CCK-8) assays.

\section{Establishment of bEnd.3 Cell Lines That Stably Express RFP-GFP-LC3 Adenovirus}

Endothelial bEnd.3 cells at a density of $80 \%$ were transfected with RFP-GFP-LC3 adenovirus diluted in serum-free DMEM. Then, we changed the medium to DMEM with $10 \%$ FBS after $6-8 \mathrm{~h}$. After 3 days, $2 \mu \mathrm{g} / \mathrm{ml}$ puromycin was added to the adenovirus transfection and control wells. After 5 days, monoclonal screening of transfected cells was performed.

\section{Measurements of Monolayer Endothelial Permeability}

The trans-endothelial electrical resistance (TEER) and paracellular permeability were measured to reflect the barrier property of the endothelial monolayer as previously described (Yang et al., 2020). In brief, wild-type or Claudin- $5^{112 \Delta 5}$-mutated bEnd. 3 cells were cultured on the trans-well plate of $0.4 \mathrm{~mm}$ pore size (Millipore, United States) for a 5 days. Then, the resistance of inserts was monitored by CellZscopeR-System (NanoAnalytics $\mathrm{GmbH}$, Muenster, Germany). TEER value was calculated as $\Omega^{*} \mathrm{~cm}^{2}$. For paracellular permeability measurement, the tightness of wild-type or Claudin- $5^{112 \Delta 5}$-mutated bEnd.3 monolayer cell was determined by the infiltration of FITC-labeled dextran $(10 \mathrm{kDa}, 0.5 \mathrm{mg} / \mathrm{L}$, Thermo-Fisher, United States). After applying the dextran inside the well, the dye in the lower chamber was measured with a spectrophotometer-computer interfaced system (BioTek Epoch, United States) at a wave length of $594 \mathrm{~nm}$. For the measurements of TEER and permeation of tracers, three repeats of each measurement were investigated for each line.

\section{Claudin-5 Rescue Assay in bEnd.3 Cells}

Claudin- $5^{112 \Delta 5}$-mutated bEnd.3 cells at a density of $80 \%$ were transfected with PIRES-eGFP or PIRES-eGFP-Claudin-5 according to the instructions of the Lipofectamine 2000 DNA transfection reagent (Invitrogen, 11668027). Then, we changed the medium to DMEM with $10 \%$ FBS after 6-8h. After $48 \mathrm{~h}$, the cells were stimulated with hypoxia for $4 \mathrm{~h}$, and the protein expression levels of Claudin-5, LC3 or inducible nitric oxide synthase (iNOS) were evaluated by immunostaining.

\section{Membrane and Cytosolic Protein Extraction Assay}

Brain microvascular endothelial cells were cultured to form a confluent monolayer in a $10 \mathrm{~cm}$ dish. Then the cells were 
exposed to hypoxic conditions for 4 and $6 \mathrm{~h}$, and we used a cell membrane and cytoplasm extraction kit (Beyotime, P0033). In brief, the cells were washed two times with cold PBS, and $1 \mathrm{ml}$ of membrane protein extraction reagent $\mathrm{A}$ was added. PMSF was added within a few minutes before use to a final concentration of $1 \mathrm{mM}$. The cells were collected in a $1.5 \mathrm{ml}$ EP tube and placed on ice for $10-15 \mathrm{~min}$. Ultrasonic crushing was performed twice at $0.6 \mathrm{~Hz}$ for $4 \mathrm{~s} /$ time. The samples were centrifuged at $700 \mathrm{~g}$ for $10 \mathrm{~min}$ at $4^{\circ} \mathrm{C}$, and the supernatant was collected and centrifuged at $14,000 \mathrm{~g}$ for $30 \mathrm{~min}$ at $4^{\circ} \mathrm{C}$. The supernatant contained the cytoplasmic proteins, which were stored at $-80^{\circ} \mathrm{C}$. The supernatant contained $30-50 \mu \mathrm{l}$ of supernatant residue to avoid contact with the precipitate. The samples were centrifuged at $4^{\circ} \mathrm{C}$ and $14,000 \mathrm{~g}$ for $10 \mathrm{~s}$. Then, $40 \mu \mathrm{l}$ of membrane protein extraction reagent $\mathrm{B}$ was added to the precipitate, vortexed for $5 \mathrm{~s}$, and placed in an ice bath for $5-10 \mathrm{~min}$, and the previous vortex and ice bath incubation steps were repeated 1-2 times. The samples were centrifuged at $14,000 \mathrm{~g}$ for $5 \mathrm{~min}$ at $4^{\circ} \mathrm{C}$, and the supernatant was collected to obtain the cell membrane protein fraction.

\section{Immunofluorescence Assay}

The cells were cultured to form a confluent monolayer in a 24-well plate. The cells were washed twice with cold PBS, and cold acetone was added and incubated for $10 \mathrm{~min}$. Then, the cells were washed once in cold 95\% ethanol, once in cold $75 \%$ ethanol, and twice with PBS, after which the cells were blocked with blocking solution at room temperature for $1 \mathrm{~h}$ (blocking solution: $1 \%$ BSA $+0.5 \%$ Tween-20 in PBS). Then, the cells were incubated with the following primary antibodies at $4^{\circ} \mathrm{C}$ overnight (1:200, diluted with blocking solution): Claudin-5 (1:200; Invitrogen, 35-2,500), LC3A/B (1:200; Cell Signaling Technology, 4,108), Caveolin-1 (1:200; Santa Cruz Biotechnology, sc-894), hypoxia inducible factor 1 subunit alpha (HIF-1a; 1:200; ProteinTech, 20960-1-AP), and iNOS (1:100; ProteinTech, 18985-1-AP). The cells were then incubated with the following secondary antibodies at room temperature for $2 \mathrm{~h}$ : Alexa Fluor 488-conjugated goat anti-mouse IgG (1:400; Thermo Fisher Scientific, A-11029) and Alexa Fluor 647-conjugated goat antirabbit IgG (1:400; Jackson ImmunoResearch, 111-605-003). DAPI (1:500; Sigma Aldrich, D9542) staining was also performed. For zebrafish tissue immunostaining, the brain sections were incubated with primary antibodies against Claudin-5 (Invitrogen, 35-2,500) and LC3B (Sigma-Aldrich, L7543), Alexa Fluor 647-conjugated goat anti-mouse IgG and Cy3-conjugated goat anti-rabbit IgG secondary antibodies and DAPI. Images were obtained with an Olympus FV3000 confocal laser microscope. Fluorescence quantitative analysis was performed using Image-Pro Plus software.

\section{Western Blotting}

Western blotting was performed as described previously (Yu et al., 2019). In brief, protein samples were separated in 10-15\% SDS-PAGE acrylamide gels, transferred onto PVDF membranes (Millipore, $0.22 \mu \mathrm{m}$ and blocked with $5 \%$ skim milk for $2 \mathrm{~h}$ ). The blots were then incubated at $4^{\circ} \mathrm{C}$ overnight with the following primary antibodies: Claudin-5 (1:1,000; Invitrogen, 34-1,600), LC3 (1:1,000; ProteinTech, 14600-1-AP), Caveolin-1 (1:200; Santa Cruz Biotechnology, sc-894), HIF-1a (1:1000; ProteinTech, 209601-AP), iNOS (1:1,000; ProteinTech, 18985-1-AP), LAMP-1 (1:1000; ProteinTech, 21997-1-AP), ATP1A1 (1:1,000, ProteinTech, 144181 -AP), or $\beta$-actin (1:1,000; Servicebio, GB12001). Then, the blots were incubated at room temperature for $2 \mathrm{~h}$ with HRP conjugated anti-rabbit or anti-mouse IgG $(\mathrm{H}+\mathrm{L} ; 1: 1,000$; Servicebio) secondary antibodies. Western blot bands were analyzed by adding ECL advance Western blotting detection reagents (Thermo, 34,580) and imaged using a SmartChemi-500 imaging system (Sage Creation Science, China). The immunoblot bands were quantitatively analyzed by ImageJ software.

\section{Flow Cytometry Analysis}

Intracellular ROS were measured using 2',7'-dichlorofluorescein diacetate (DCFH-DA, Beyotime, S0033S). DCFH-DA was diluted with serum-free medium to a final concentration of $10 \mu \mathrm{M}$. The cells were harvested in a $15 \mathrm{ml}$ centrifuge tube, and $1 \mathrm{ml}$ of diluted DCFH-DA was added to the cells and incubated for $20 \mathrm{~min}$ at $37^{\circ} \mathrm{C}$. The cells were washed three times with serum-free DMEM. Then, detection was performed by flow cytometry. Cell apoptosis was detected by Annexin V-FITC cell apoptosis detection kit (Beyotime, C1062M). Briefly, cells were harvested and stained with annexin V-FITC and propidium iodide for $20 \mathrm{~min}$ at room temperature and examined using a flow cytometer.

\section{Statistical Analysis}

For statistical analysis, GraphPad Prism 5 software was used. All values are presented as the means $\pm S D$, and two-tailed Student's $t$-test or one-way ANOVA was applied to determine statistical significance $\left({ }^{*} p<0.05,{ }^{* *} p<0.01\right.$ and $\left.{ }^{* * *} p<0.005\right)$.

\section{RESULTS}

\section{Involvement of Endothelial Claudin-5 in Autophagy Under Hypoxic Condition}

Our previous study has shown the activation of autophagy in endothelial cells in response to hypoxia (Yang et al., 2019b, 2020). To confirm the alterations of autophagic flux in bEnd.3 cells in response to hypoxia, bEnd.3 cells were transfected with RFP-GFP-LC3 adenovirus for live imaging analysis. As shown in Supplementary Figure S1, hypoxia induction increased the numbers of both GFP and RFP dots in bEnd.3 cells compared with that in normoxia-treated cells, and we found that the $\mathrm{red} /$ yellow dot ratio increased due to hypoxia induction, indicating an increased expression of LC3, a marker of autophagy, and a formation of autophagosomes to autolysosomes in hypoxiatreated cells. Previous studies have shown that short-term hypoxia induction causes the redistribution and endocytosis of membranous Claudin-5, and autophagy is then activated to mediate the degradation of abnormally accumulated Claudin- 5 in cytosol, thereby eliminating ROS and protecting the integrity of the BBB barrier from fast injury (Yang et al., 2019b, 2020). 


\section{The Impact of Claudin-5 on the Activation of Autophagy Under Early Hypoxia Induction}

To explore the impact of the membranous TJ protein Claudin-5 on the occurrence of autophagy in response to hypoxia, two stabilized Claudin-5-knocked out bEnd.3 mutated cell lines, Claudin- $5^{112 \Delta 5}$ with a 5 bp deletion and Claudin- $5^{297 \Delta 4}$ with a $4 \mathrm{bp}$ deletion, were first constructed and generated by CRISPR/ Cas9 strategy (Supplementary Figure S2A). The expression of Claudin-5 was examined by immunofluorescence staining and immunoblotting respectively, and the results indicated a total loss of Claudin-5 from cell membrane in these lines (Supplementary Figures S2B,C). To verify the endothelial barrier function of bEnd.3 cells after loss of Claudin-5, the TEER and paracellular permeability of the wild-type or Claudin $-5^{112 \Delta 5}$ mutated bEnd.3 monolayer cells were measured. The results indicated a significant loss of the tightness of monolayer Claudin- $5^{112 \Delta 5}$ mutated cells in comparison to that of the wild-type bEnd.3 cells (Supplementary Figures S2D,C). Since a functional alteration was observed in bEnd. 3 cells after loss of Claudin-5, we additionally analyzed the cell viability in response to hypoxia by CCK- 8 assays in both wild-type and mutated bEnd.3 cells. The result showed that the survival rate of both Claudin-5-knocked out cells was still above $90 \%$ till $6 \mathrm{~h}$ post-hypoxia induction, and there was no significant variations of the cell viability among the wild-type and Claudin-5-knocked out lines under short term hypoxic induction (Supplementary Figure S3).

Next, to verify whether Claudin-5 affects the occurrence of autophagy in response to hypoxia, the expression of LC3 in Claudin- $5^{\mathrm{wt}}$ and Claudin-5 $5^{112 \Delta 5}$ bEnd. 3 cells were analyzed by immunofluorescence staining first. As a result, it was found that in bEnd.3 Claudin-5 ${ }^{\mathrm{wt}}$ cells, Claudin-5 and LC3 showed obvious colocalization in the cytoplasm after 4 or $6 \mathrm{~h}$ hypoxia induction (white arrows in Figure 1A). The expression level of LC3 in cells with Claudin-5 knockout was significantly reduced (Figure 1A). Meanwhile, the expression levels of LC3 and LAMP-1, a lysosomal membrane protein and a specific marker of autolysosomes (Zhan et al., 2018), in Claudin- $5^{\mathrm{wt}}$ and Claudin- $5^{112 \Delta 5}$ bEnd.3 cells in response to hypoxia were analyzed by immunoblotting. The protein expression levels of both LC3 and LAMP-1 in bEnd. 3 Claudin- $5^{112 \Delta 5}$ cells were significantly lower than those in Claudin $-5^{\mathrm{wt}}$ bEnd. 3 cells after 4 or $6 \mathrm{~h}$ hypoxia induction (Figure 1B). The inhibition of the
A

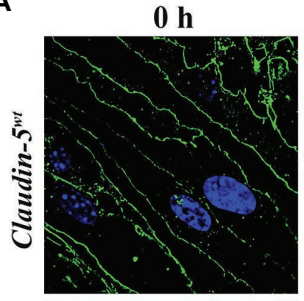

$4 \mathrm{~h}$
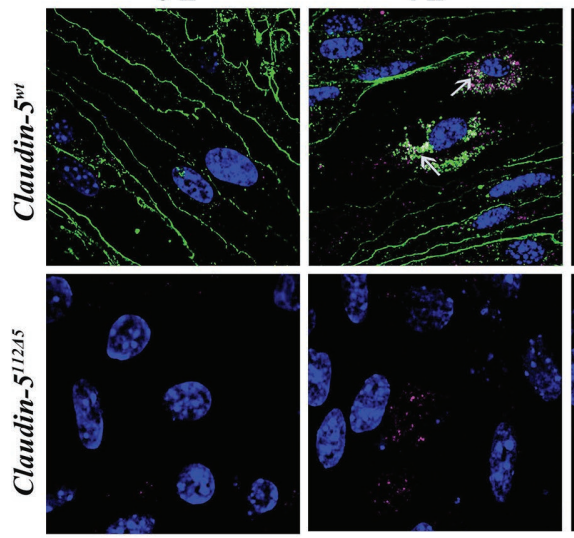

Claudin-5 LC3 $\quad$ Nuclei

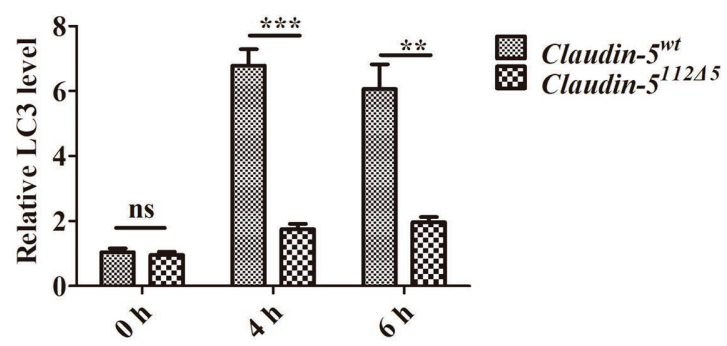

B
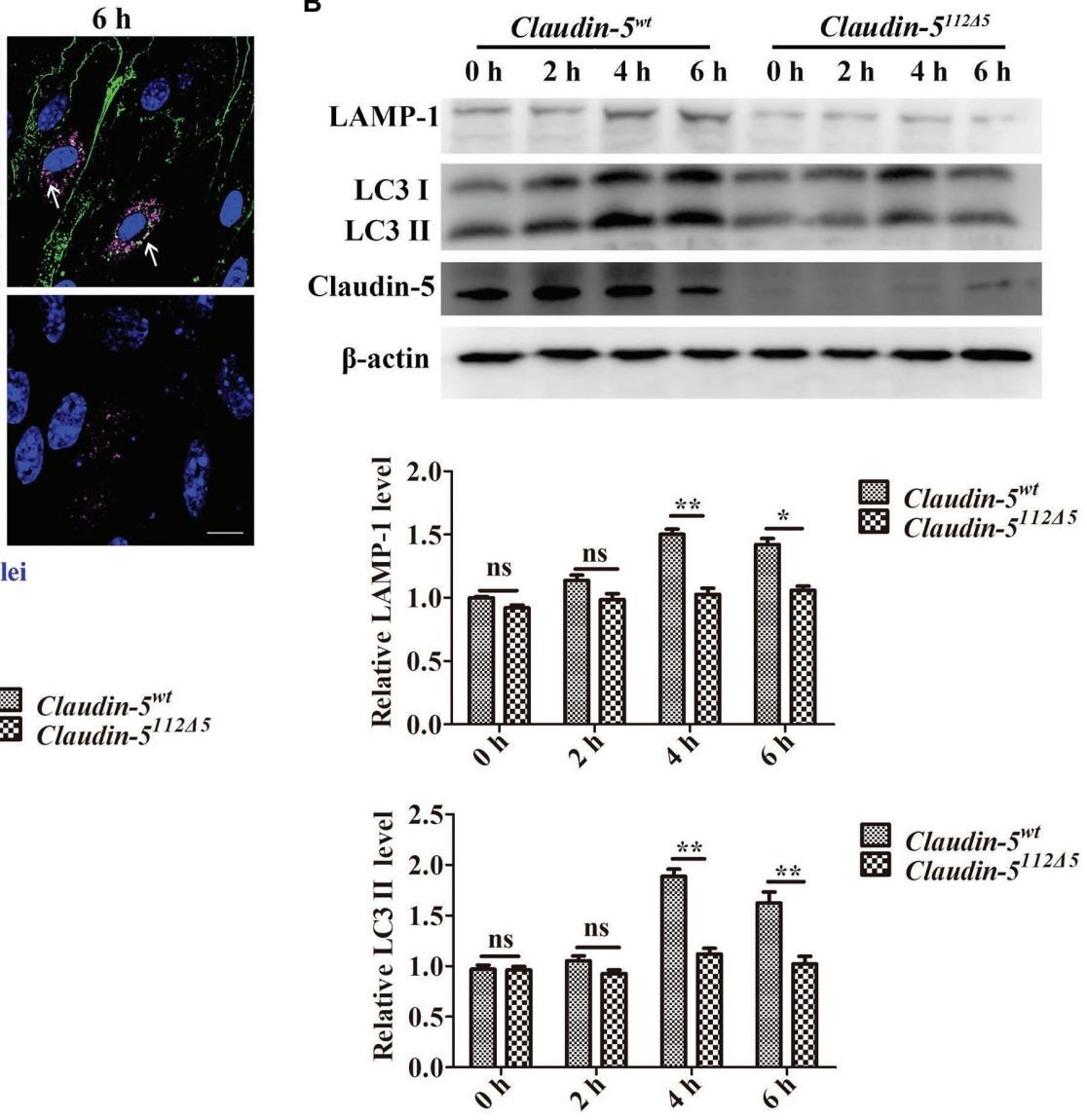

FIGURE 1 | The specificity of the involvement of Claudin-5 in autophagy under hypoxia induction. (A) Confocal microscopy images of Claudin-5 (green) and LC3 (purple). Colocalization of Claudin- 5 with LC3 (white arrow) and quantitative analysis of LC3 levels by Image-Pro Plus. Mean \pm SD, $n=3$ independent experiments per group. Scale bar, $10 \mu \mathrm{m}$. (B) Western blot analysis of the autophagy markers LC3 and LAMP-1. Mean \pm SD, $n=3$ independent experiments per group. * $p<0.05$ ${ }^{* *} p<0.01$ and ${ }^{* * *} p<0.005$. 
autophagy activation was confirmed by another Claudin-5 mutant bEnd.3 line of Claudin- $5^{297 \Delta 4}$, where the expression of both LC3 and LAMP-1 was sufficiently reduced due to the loss of Claudin-5 in response to hypoxia induction for 4 or $6 \mathrm{~h}$ (Supplementary Figure S4). A rescue experiment was also performed to further verify the specificity of Claudin-5 on hypoxia-induced autophagy occurrence. The PIRES-eGFP or pIRES-eGFP-Claudin-5 plasmid was first transfected into mutated Claudin- $5^{112 \Delta 5}$ bEnd. 3 cells. The followed immunofluorescence staining showed that cells successfully transfected with the pIRES-eGFP-Claudin-5 plasmid expressed LC3 at levels higher than the vehicle in response to $4 \mathrm{~h}$ hypoxia induction (Supplementary Figure S5). These results revealed that endothelial membranous Claudin-5 was specifically involved in the activation of autophagy during the early stage of hypoxia.

We also sought in vivo evidences supporting the correlations between Claudin- 5 and autophagy activation in endothelial cells under hypoxia. To this end, endothelial eGFP-specific transgenic
$\mathrm{Tg}(\mathrm{kdrl}: \mathrm{eGFP})$ zebrafish was applied to investigate the autophgy activation in endothelial cells (Yang et al., 2019b, 2020). Since zebrafish Claudin-5b is the main Claudin expressed in cerebrovascular endothelial cells during the embryonic stages (Yang et al., 2021), MO-mediated silencing of claudin-5b in zebrafish embryos was first performed. After 3 days, it was found that in response to $3 \mathrm{~h}$ hypoxia treatment, the control morphant embryo presented much severer brain injury than that of the claudin-5 $b$ morphants (red arrowheads, Figures 2A,B). Immunofluorescence staining with the embryonic brains further revealed that in control morphant zebrafish larvae endothelial cells, the expression level of LC3 was higher than that in the cerebrovascluar endothelial cells of claudin-5b morphants posthypoxia induction (Figures 2C,D). Above in vivo and in vivo evidences confirmed that loss of Claudin-5 could partly affect the activation of endothelial autophagy caused by hypoxia induction.

Since, we have observed a higher phenotype ratio of brain injury in control embryos in comparison to that in the brain

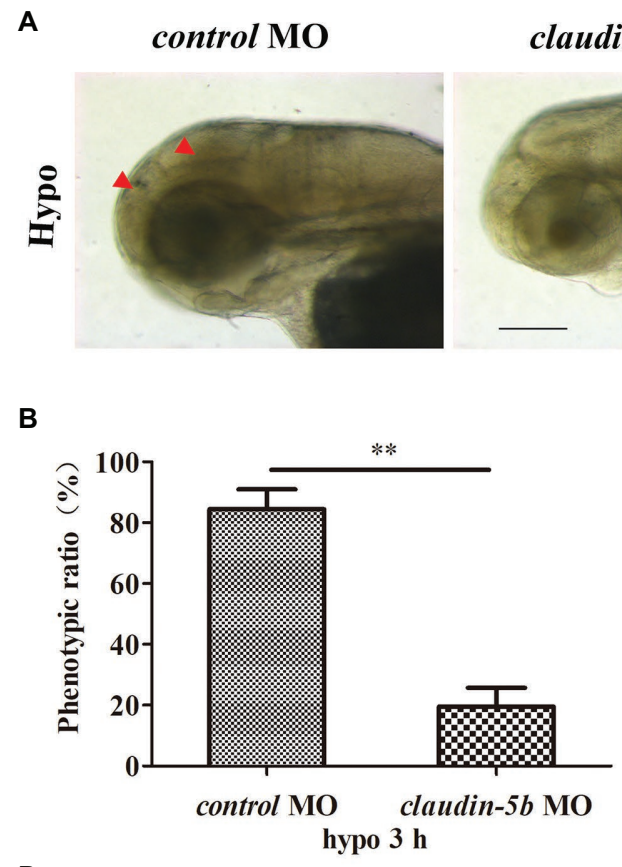

D

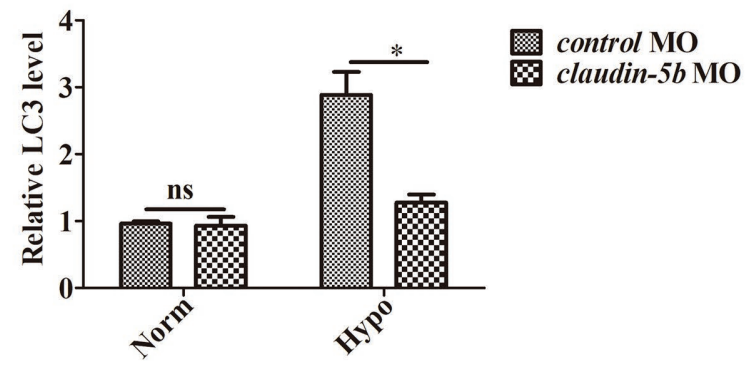

C
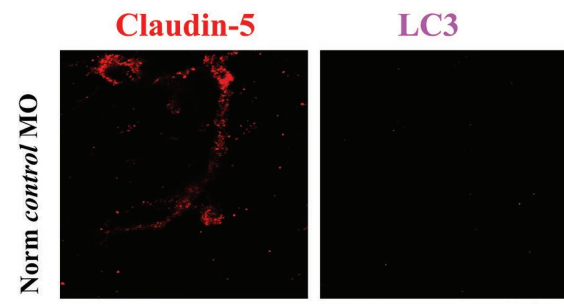

kdrl:eGFP
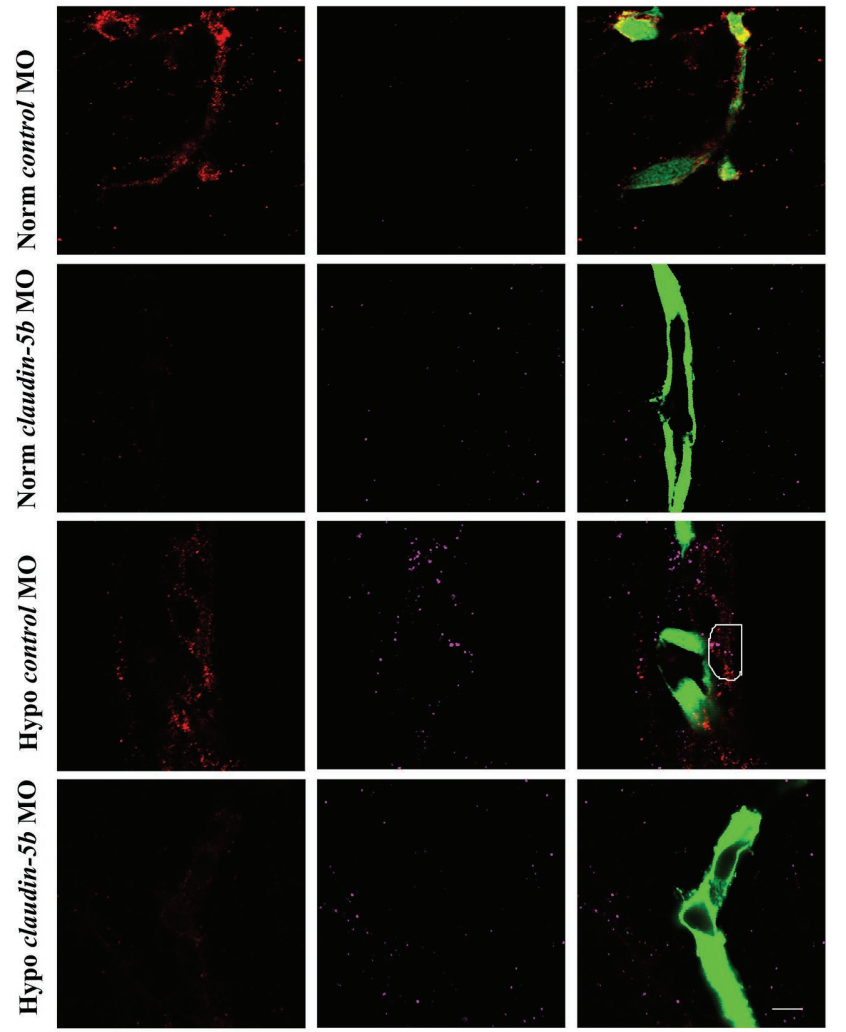

FIGURE 2 | Loss of Claudin-5b in zebrafish embryos inhibits activation of autophagy in cerebrovascular endothelial cells (ECs). Morpholino (MO) silencing of claudin-5b in endothelial eGFP-specific transgenic $\mathrm{Tg}(\mathrm{kdrl}$ :eGFP) zebrafish embryos. (A) Brain phenotype (red arrowheads) of control MO and claudin-5b MO zebrafish larvae in response to hypoxia. Scale bar, $100 \mu \mathrm{m}$. (B) Quantitative analysis of the severe injury rates of contro/ MO and claudin-5b MO zebrafish larval brains. Mean $\pm \mathrm{SD}, n=3$ independent experiments per group. (C) Confocal microscopy images of Claudin-5 (red) and LC3 (purple). Scale bar, $5 \mu \mathrm{m}$. (D) Quantitative analysis of LC3 levels by Image-Pro Plus. Mean \pm SD, $n=3$ independent experiments per group. ${ }^{*} p<0.05$ and ${ }^{* *} p<0.01$. 
of claudin-5b morphants post-hypoxia treatment, we wondered whether lack of endothelial Claudin- 5 could alleviate the cell injury or death caused by hypoxia in a certain extent. Therefore, we analyzed hypoxia-caused apoptosis of the Claudin- $5^{\mathrm{wt}}$ and Claudin-5 knocked out bEnd. 3 cells. The results indicated that, after 4 h-hypoxia induction, Claudin- $5^{\mathrm{wt}}$ bEnd. 3 cell showed a higher apoptosis ratio than Claudin- $5^{112 \Delta 5}$ or Claudin- $5^{297 \Delta 4}$ bEnd.3 cells (Supplementary Figure S6).

\section{Claudin-5 Affects Caveolae-Mediated Endocytosis Under Hypoxia Conditions}

It has been reported that in response to hypoxia induction, membranous Claudin-5 in the vascular endothelial cells is packaged by Cav-1-composed caveolae and is then endocytosed into the cytoplasm, where it is eventually degraded by autophagosomes or autolysosomes (Liu et al., 2012, 2016). Therefore, we next asked whether the expression and localization of Cav-1 in hypoxia-treated bEnd. 3 cells will be altered since Claudin- 5 is absent. To this end, an immunofluorescence staining against Cav-1 was first performed in Claudin- $5^{\mathrm{wt}}$ and Claudin $-5^{112 \Delta 5}$ bEnd. 3 cells after 4 or $6 \mathrm{~h}$ hypoxia induction. The results indicated that most of the Cav-1 still localized in the membrane of Claudin- $5^{112 \Delta 5}$ bEnd.3 cells due to the loss of Claudin-5, and that the Cav-1, which colocalized with Claudin-5 in the cytosol of Claudin- $5^{\mathrm{wt}}$ bEnd. 3 cells was much more than that in Claudin$5^{112 \Delta 5}$ bEnd. 3 cells in response to hypoxia for 4 and $6 \mathrm{~h}$ (Figure 3A). There was no significant effect of accumulation or endocytosis of Cav-1 in bEnd.3 Claudin- $5^{12 \Delta 5}$ cells in response to hypoxia for 4 and $6 \mathrm{~h}$ (Figure 3A). The protein level of Cav-1 in the cell membrane and cytoplasm was also quantified by immunoblotting analyses. The results confirmed that due to lack of Claudin-5, there was almost no loss of membranous Cav-1 post-hypoxia induction for $6 \mathrm{~h}$, and there was no significant accumulation of Cav-1 in the cytoplasm either in comparison with that of the Claudin- $5^{\mathrm{wt}}$ bEnd.3 cells (Figure 3B). These results confirmed that $\mathrm{Cav}-1$ is mainly responsible for the endocytosis of membranous Claudin-5 in response to hypoxia induction, while in the absence of Claudin-5, the delocalization of Cav-1 from endothelial membrane is sufficiently inhibited.

\section{The Effects of Endothelial Claudin-5 on the Expression of iNOS and HIF-1a Under Hypoxia}

Previous studies have shown that iNOS-derived NO is the main cause of Cav-1-mediated Claudin-5 ectopic activity in response to hypoxia (Liu et al., 2016; Yang et al., 2019a). HIF-1a is a transcriptional regulator produced by the body during hypoxia, and HIF-1a is one of the main factors that

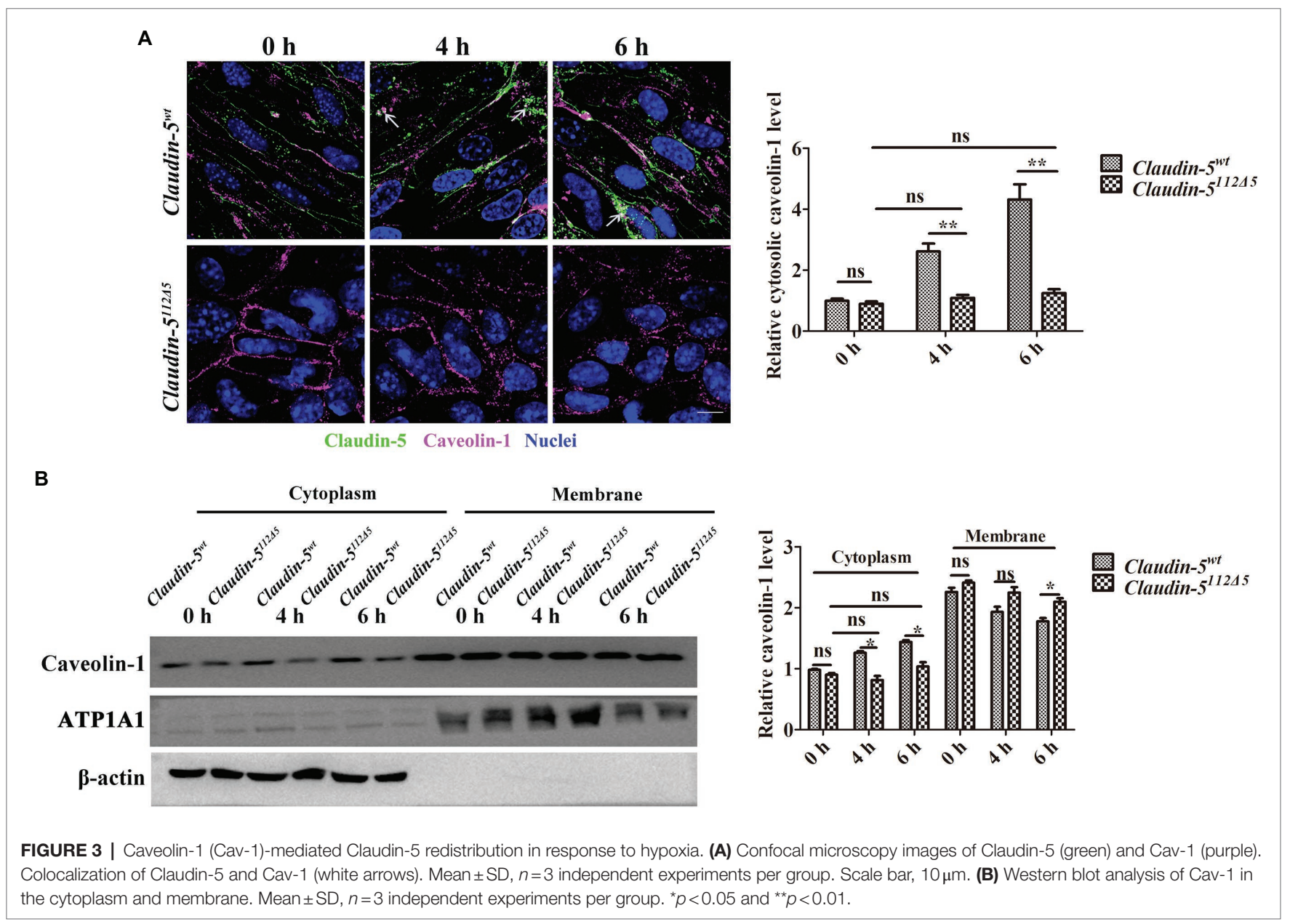


regulates iNOS transcription (Robinson et al., 2011). Therefore, to ask whether the loss of Claudin- 5 could conversely affect the production of iNOS and its upstream regulator of HIF-1a, we next analyzed the their expression levels in both wild-type and Claudin-5 mutant bEnd. 3 cells after 4 or $6 \mathrm{~h}$ hypoxia induction by immunofluorescence staining and immunoblotting. We found that the expressions of both iNOS and HIF-1a in Claudin-5 mutant bEnd. 3 cells were significantly lower than that in Claudin- $5^{\mathrm{wt}}$ cells in response to hypoxia induction for 4 and $6 \mathrm{~h}$ (Figures $4 \mathbf{A}, \mathbf{B}$ ). To confirm the specificity of membranous Claudin-5 on endothelial iNOS generation under hypoxia, rescue experiments by transfecting pIRES-eGFPClaudin-5 plasmid in Claudin-5 mutant bEnd.3 cells were performed. It was found that restored expression of Claudin-5 in bEnd.3 Claudin- $5^{112 \Delta 5}$ mutant cells could recover the generation of iNOS in response to hypoxia treatment for $4 \mathrm{~h}$ (Supplementary Figure S7). Immunoblotting analyses also revealed that the protein expression levels of HIF-1a and iNOS in wild-type bEnd. 3 cells after 4 and $6 \mathrm{~h}$ of hypoxia were higher than that in Claudin-5 $5^{112 \Delta 5}$ and Claudin-5 ${ }^{297 \Delta 4}$ bEnd.3 cells (Figure 4C), indicating that lack of membranous Claudin-5 could partly inhibited the production of endothelial HIF-1a and iNOS under short term hypoxia induction.

\section{ROS Generation Was Affected by Claudin- 5 in bEnd.3 Cells Post-hypoxia Induction}

ROS are produced by various sources in the CNS, including mitochondria, NADPH oxidase, and NOS, especially under hypoxia conditions (Yamamoto et al., 2006; Kuthati et al., 2019). We used DCFH-DA to measure intracellular ROS and then analyzed these levels by flow cytometry. It was found that the ROS level in Claudin- $5^{\text {wt }}$ bEnd.3 cells was higher than those in both Claudin- $5^{12 \Delta 5}$ and Claudin- $5^{297 \Delta 4}$ mutant bEnd.3 cells in response to hypoxia induction for 4 and $6 \mathrm{~h}$, respectively (Figure 5A). This also suggests that the production of cellular ROS may be directly related to NOS in response to hypoxia. Previous studies have shown that under hypoxia induction, ROS could function on driving the expression of Bnip3 (Bcl-2 and adenovirus E1B 19-kDa interacting protein 3), which is a single transmembrane protein mediating autophagy to maintain cell survival (Zhang et al., 2019). Hence, immunoblotting analysis was performed to detect the protein expression level of Bnip3 in Claudin-5 mutated bEnd. 3 cells. The results revealed that in comparison with the expression in wild-type control bEnd.3 cells, the expression level of Bnip3 in Claudin- $5^{112 \Delta 5}$ bEnd.3 cells was significantly lower after 4 or $6 \mathrm{~h}$ hypoxia induction (Figure 5B). Above results indicated that the existence of membranous Claudin-5 affects the production of ROS and the downstream Bnip3 expression in response to early hypoxia induction.

In summary, based on our previous finding that under early hypoxia injury, autophagy protects the BBB from fast breakdown by regulating the dynamics of Claudin- 5 , we further revealed the existence of membranous Claudin- 5 could conversely affect the occurrence of autophagy in endothelial cells by both in vivo and in vitro evidences (Figure 5C). This is probably due to the attenuated endocytosis of caveolae-packaged Claudin-5 and reduced autophagic degradation of abnormally accumulated proteins in cytosol during the early hypoxia induction stages (as early as $6 \mathrm{~h}$ ). Meanwhile, our results indicate that lack of endothelial Claudin-5 caused a decreased sensitivity and production of HIF-1a and iNOS to early hypoxia induction, which together with the downstream ROS and Bnip3, affect the endothelial autophagy activation.

\section{DISCUSSION}

Our previous have shown that under early stage of starvation/ hypoxia induction, endothelial membranous Claudin-5 was endocytosed from the cell membrane into the cytoplasm (Yang et al., 2019b, 2020). Additionally, we also revealed that endothelial autophagy functions on the clear of the abnormally accumulated cytosolic Claudin-5 and caveolin-1, therefore, protects endothelial cell from apoptosis and maintains the integrity of the endothelial barrier (Yang et al., 2019b, 2020). In this study, it is found that the activation of autophagy was partly inhibited in Claudin-5 knocked out bEnd.3 cells, indicating membranous TJ protein of Claudin-5 could conversely affect the endothelial autophagy under hypoxia conditions. Therefore, two questions were raised regarding (1) the specific involvement of Claudin-5 in autophagy activation and (2) the mechanism by which Claudin- 5 affects endothelial autophagy in the early stage of hypoxia treatment.

To identify the correlations between Claudin- 5 and autophagy activation, two stable Claudin-5 knockout mutant cell lines (bEnd.3 Claudin-5 $5^{12 \Delta 5}$ and bEnd.3 Claudin-5 ${ }^{297 \Delta 4}$ ) were successfully constructed, respectively, by CRISPR/Cas9 strategy in this study. Meanwhile, the endothelial claudin-5b knocked down zebrafish embryo was used as in vivo model to identify the activation of autophagy under hypoxia conditions. Both in vivo and in vitro evidences confirmed the findings that the membrane expression of Claudin-5 affects the occurrence of endothelial autophagy in response to short-term hypoxia induction. We further explored the underlying mechanism by which Claudin-5 is involved in autophagy activation during early stage of hypoxia treatment. We have shown that cerebral ischemia can cause Cav-1-mediated redistribution and endocytosis of Claudin-5, which causes BBB disruption in the early stages of stroke (Yang et al., 2020). Meanwhile, previous studies have shown that knock down of Cav-1 completely eliminates the redistribution of Claudin- 5 induced by hypoxia and partially prevents the destruction of the BBB (Liu et al., 2016), indicating a tight correlation of membranous Cav-1 and Claudin-5, and a necessity of Cav-1 in membranous Claudin-5 endocytosis. Combining our findings with reduced protein expressions of cytoplasmic Cav-1 in bEnd.3 Claudin$5^{112 \Delta 5}$ cells compared with that in bEnd. 3 Claudin $-5^{\text {wt }}$ cells under hypoxia, we conclude that absent of membranous/ endocytosed Claudin- 5 and reduced Cav- 1 in cytosol suppresses the activation of endothelial autophagy under hypoxia induction due to the lack of cytosolic substrates for the autophagic degradation (Figure 5C). 
A
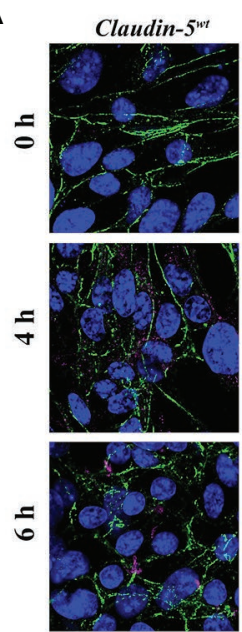

Claudin-5 iNOS Nuclei

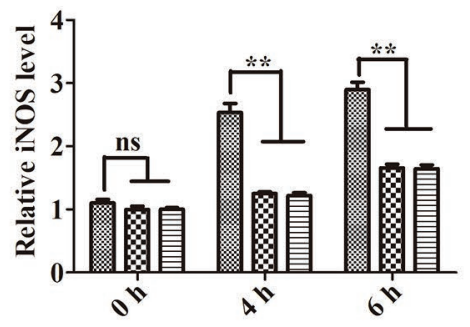

B
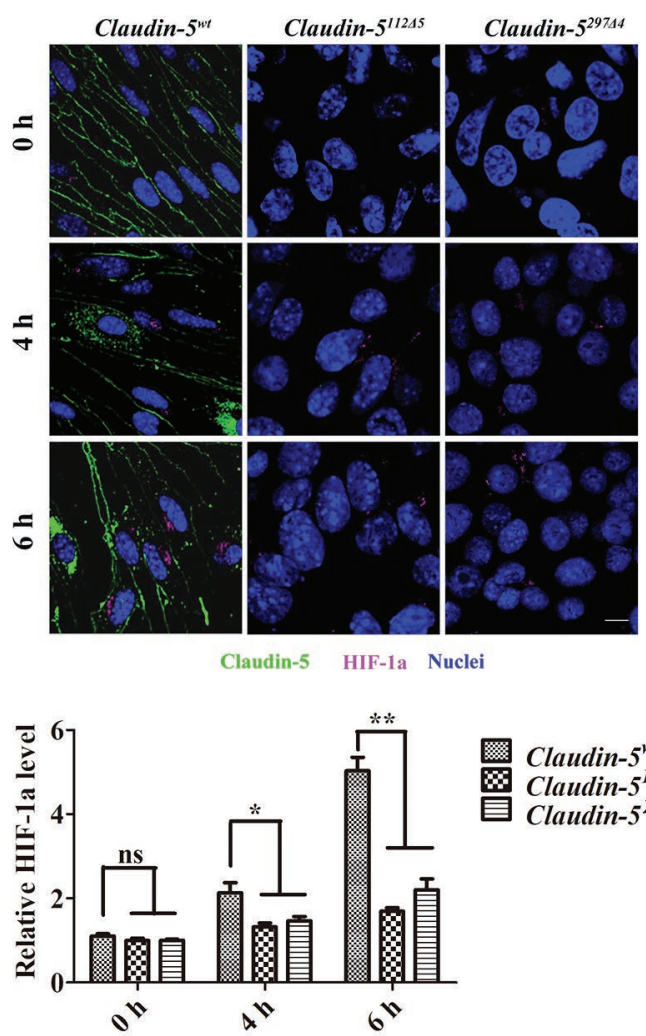

(1audin-5 $5^{\text {wt }}$ Claudin-5 $-5^{11245}$

C
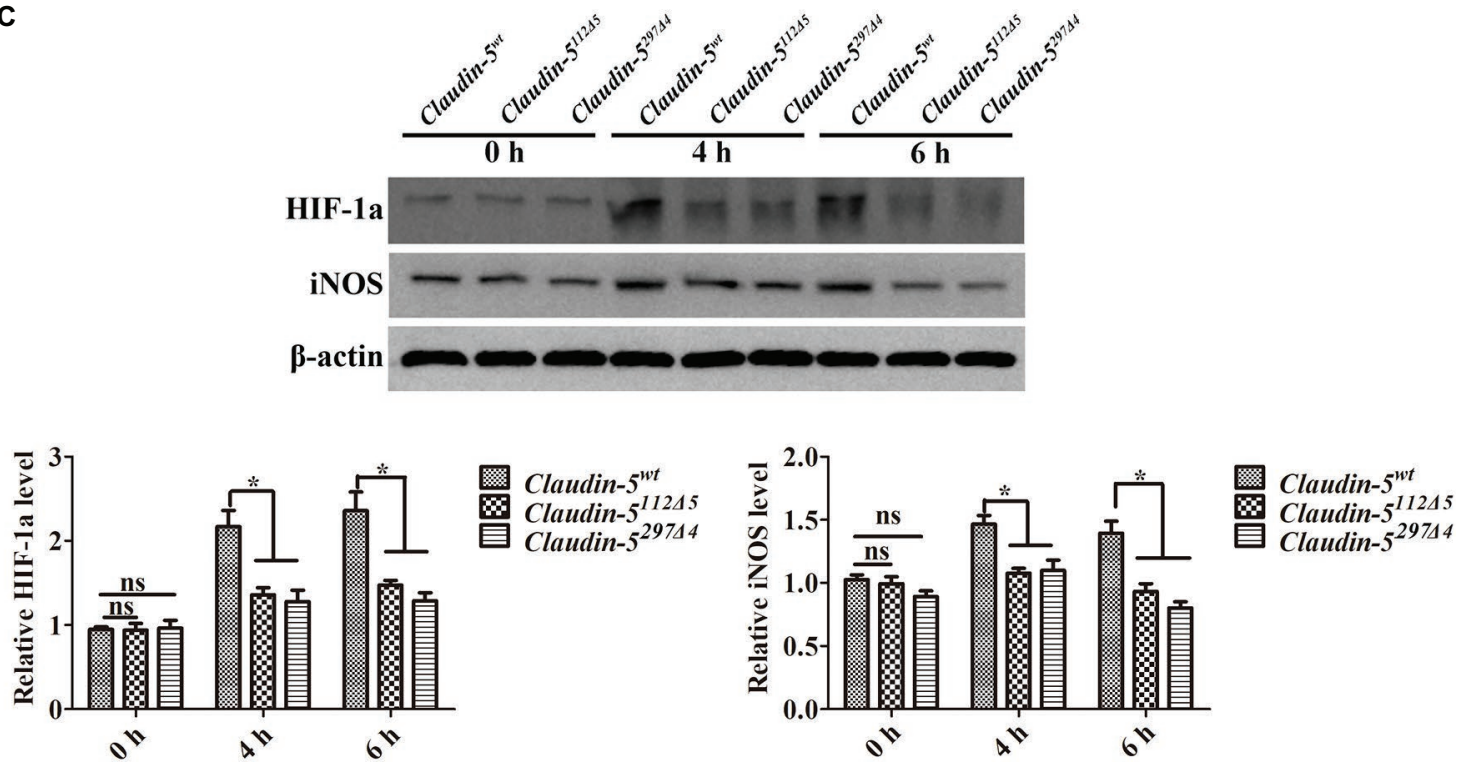

FIGURE 4 | Inducible nitric oxide synthase (iNOS) induces Cav-1-mediated Claudin-5 translocation in response to hypoxia. (A,B) Confocal microscopy images of Claudin-5 (green), iNOS (purple), and hypoxia inducible factor 1 subunit alpha (HIF-1a; purple). Scale bar, $10 \mu \mathrm{m}$. Mean \pm SD, $n=3$ independent experiments per group. (C) Western blot analysis of iNOS and HIF-1a. Mean $\pm S D, n=3$ independent experiments per group. ${ }^{*} p<0.05$ and ${ }^{* *} p<0.01$.

Liu et al. (2016) has reported that nitric oxide (NO) produced by iNOS could also induce Cav-1-mediated Claudin-5 redistribution in response to hypoxia. NOS has three types: neuronal (nNOS or NOS1), endothelial (eNOS or NOS3), and inducible (iNOS or NOS2). These NOS subtypes increase after cerebral ischemia. iNOS is different from eNOS and nNOS, and iNOS produces a large amount of $\mathrm{NO}$ ( $\mu \mathrm{M}$ to $\mathrm{mM}$ range) in response to various stimuli such as LPS, cytokines, or 


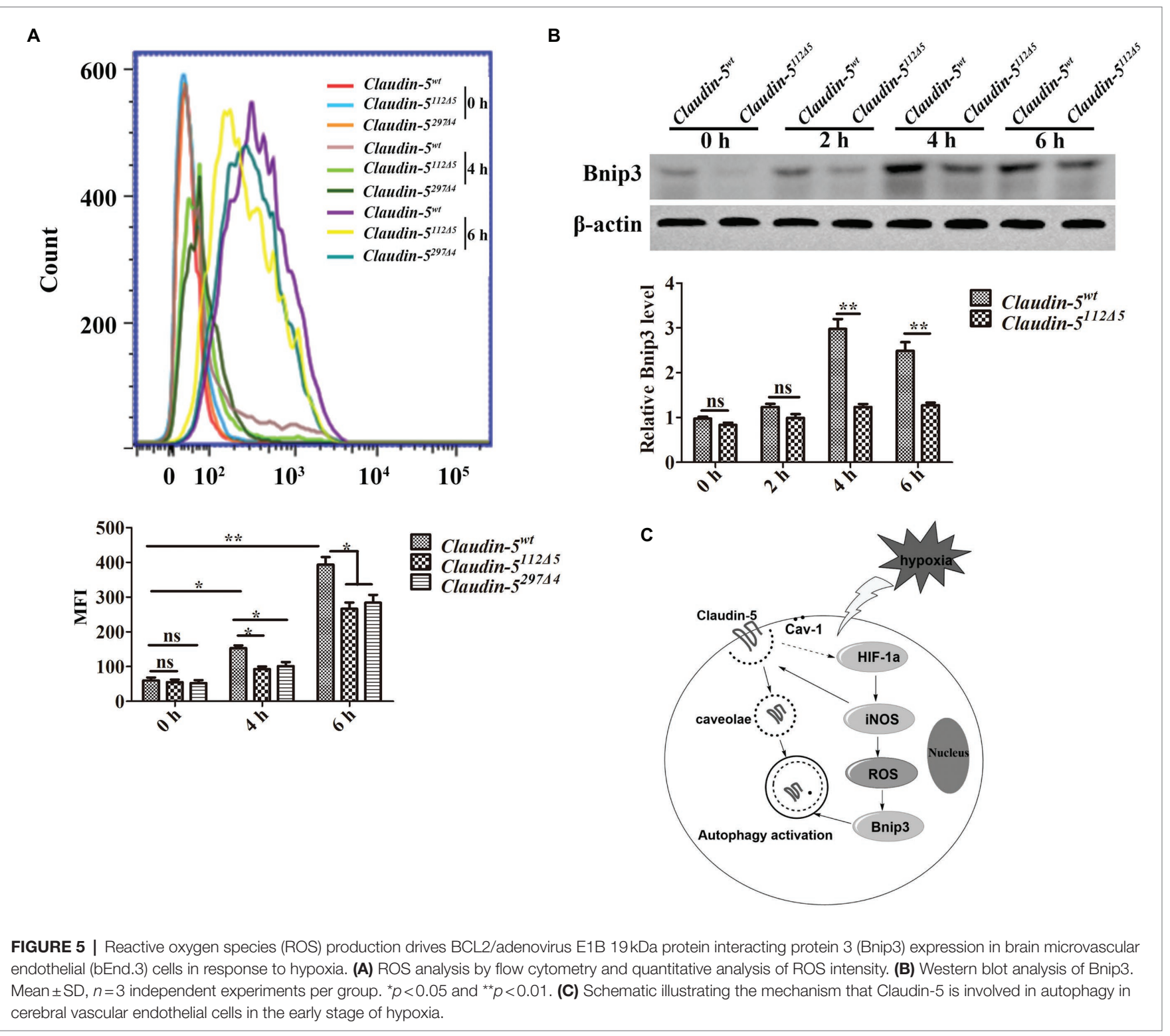

hypoxia/ischemia (Kirk et al., 1990; Lapointe et al., 2006; Lakhan et al., 2009; Costa et al., 2016). In this study, the iNOS expression level in Claudin-5 $5^{112 \Delta 5}$ and Claudin- $5^{297 \Delta 4}$ mutant bEnd. 3 cells was much lower than that in Claudin- $5^{\text {wt }}$ bEnd. 3 cells in response to hypoxia as shown by immunofluorescence staining and immunoblotting analyses. HIF-1a, as a transcriptional regulator produced most of cells during hypoxic injury, is one of the main regulators of iNOS transcription (Nizet and Johnson, 2009; Takeda et al., 2010). Our data showed that after short period hypoxia induction, the expression level of HIF-1a in Claudin-5 $5^{112 \Delta 5}$ and Claudin-5 $5^{297 \Delta 4}$ mutant bEnd.3 cells was significantly lower than that in Claudin $-5^{\text {wt }}$ bEnd. 3 cells. As a downstream effecter of iNOS, ROS is usually produced by various sources in the CNS, including mitochondria, NADPH oxidase, and NOS (Galley, 2011; Chan and Chan, 2014; Steinz et al., 2020). We used DCFH-DA to measure the ROS level in endothelial cells, and the data showed that the ROS generation in Claudin- $5^{\mathrm{wt}}$ bEnd.3 cells were higher than those in Claudin$5^{112 \Delta 5}$ and Claudin- $5^{297 \Delta 4}$ bEnd. 3 cells in response to early hypoxia stimulation. Previous studies have shown that ROS could drive the expression of downstream Bnip3 and promote the occurrence of autophagy in response to hypoxia (Xu et al., 2015; Zhang et al., 2019). Additionally, we analyzed the Bnip3 expression in Claudin- $5^{112 \Delta 5}$ bEnd. 3 cells, and the immunoblotting analyses showed that loss of Claudin-5 efficiently inhibited Bnip3 expression in hypoxia-treated endothelial cells. This probably also suppresses the activation of autophagy to a certain degree. We speculate that the existence of membranous Claudin- 5 affects the HIF-1a/iNOS/ROS/Bnip3 pathway might be due to a reduced sensitivity of Claudin-5-mutated endothelial cells to the short term hypoxia stimulation. On the other hand, we have reported in our previous studies that autophagy is responsible for the degradation of cytosolic Claudin-5 induced by early hypoxia treatment (shorter than 12h), while only under 
long-period hypoxia induction (24h), TJ protein of ZO-1 is selectively degraded by autophagy (Yang et al., 2020). Besides, the degradation of another TJ protein of Occludin is depending on the ubiquitin/proteasome system instead of autophagic pathway (Traweger et al., 2002). Therefore, it is possible that autophagy is mainly responsible for the specific degradation of endocytosed Claudin-5, and the repression of endothelial autophagy during early hypoxia induction is due to lack of delocalized cytosolic Claudin-5 to degrade.

As an important membranous TJ protein, endothelial Claudin-5 functions on maintain the integrity and tightness of endothelial barriers. Lack of Claudin-5 in cerebral endothelial cells causes a leakage of BBB (Nitta et al., 2003). Here, although mutation/ loss of Claudin- 5 in bEnd. 3 cells caused a decrease of monolayer endothelial cell barrier function, it showed no affect on the cell viability even under the hypoxia conditions for longer than $6 \mathrm{~h}$. Moreover, it is surprising to find in our study that under early hypoxia induction, absence of membranous Claudin- 5 could increase the resistance of endothelial cell apoptosis to the hypoxic injury, irrespective of the loss of its endothelial barrier functions. The hypoxia treatment assay on zebrafish embryos confirmed that knockdown of endothelial claudin-5 in the vascular endothelial cells is beneficial to the survival ratio of CNS cells. This might be because in the endothelial cells missing membranous Claudin-5, there is weak cytotoxicity due to decreased accumulations of abnormal proteins in cytosol, which helps with the cell survive from the hypoxic injury.

In summary, this study reveals a previously unknown but essential function of membranous Claudin-5 on activating autophagy in cerebrovascular endothelial cells during early stage of hypoxia induction. Combing with the previous reports that autophagy is able to mediate the degradation of cytosolic Claudin-5 to protect endothelial barrier from fast injury under hypoxia treatment, our findings reveal a bidirectional regulatory mechanisms of TJ protein Claudin-5 and endothelial autophagy under hypoxic conditions. These studies may provide theoretical basis for clarifying the mechanism of BBB injury and also potential emergency protection mechanisms in stroke.

\section{REFERENCES}

Chan, S. H., and Chan, J. Y. (2014). Brain stem NOS and ROS in neural mechanisms of hypertension. Antioxid. Redox Signal. 20, 146-163. doi: 10.1089/ars.2013.5230

Costa, E. D., Rezende, B. A., Cortes, S. F., and Lemos, V. S. (2016). Neuronal nitric oxide synthase in vascular physiology and diseases. Front. Physiol. 7:206. doi: 10.3389/fphys.2016.00206

Feng, J., Chen, X., and Shen, J. (2017). Reactive nitrogen species as therapeutic targets for autophagy: implication for ischemic stroke. Expert Opin. Ther. Targets 21, 305-317. doi: 10.1080/14728222.2017.1281250

Galley, H. F. (2011). Oxidative stress and mitochondrial dysfunction in sepsis. Br. J. Anaesth. 107, 57-64. doi: 10.1093/bja/aer093

Gholami, L., Jokar, S., Fatahi, Y., Samandari, H., Almaki, J. H., Hosseini, M., et al. (2020). Targeting caveolin-1 and claudin-5 with AY9944, improve blood-brain barrier permeability; computational simulation and experimental study. Cell. Mol. Neurobiol. doi: 10.1007/s10571-020-01004-z [Epub ahead of print]

Greene, C., Hanley, N., and Campbell, M. (2019). Claudin-5: gatekeeper of neurological function. Fluids Barriers CNS 16:3. doi: 10.1186/s12987-019-0123-Z

\section{DATA AVAILABILITY STATEMENT}

The original contributions presented in the study are included in the article/Supplementary Material, further inquiries can be directed to the corresponding author.

\section{ETHICS STATEMENT}

The animal study was reviewed and approved by Guangdong Medical University. Handling of zebrafish was performed in accordance with Guangdong State Regulations on Laboratory Animal Management.

\section{AUTHOR CONTRIBUTIONS}

PY performed the experiments, analyzed the data, and wrote the manuscript. YL and GZ generated the mutated cell lines and performed the in vivo experiments. $\mathrm{WL}$ and $\mathrm{BC}$ gave extract suggestions on this study. JZ initiated the study, designed the experiments, analyzed the data, and wrote the manuscript. All authors contributed to the article and approved the submitted version.

\section{FUNDING}

This work was supported by the National Natural Science Foundation of China (Grant Nos. 31771628 and 31970777), the Special and Innovative Projects of Guangdong High Schools (2018KTSCX080) to JZ, and the Initial Research Funding of Guangdong Medical University (2XK20019) to PY.

\section{SUPPLEMENTARY MATERIAL}

The Supplementary Material for this article can be found online at: https://www.frontiersin.org/articles/10.3389/fphys.2021.737474/ full\#supplementary-material

Haruwaka, K., Ikegami, A., Tachibana, Y., Ohno, N., Konishi, H., Hashimoto, A., et al. (2019). Dual microglia effects on blood brain barrier permeability induced by systemic inflammation. Nat. Commun. 10:5816. doi: 10.1038/ s41467-019-13812-z

Hashimoto, Y., and Campbell, M. (2020). Tight junction modulation at the blood-brain barrier: current and future perspectives. Biochim. Biophys. Acta Biomembr. 1862:183298. doi: 10.1016/j.bbamem.2020.183298

Kadry, H., Noorani, B., and Cucullo, L. (2020). A blood-brain barrier overview on structure, function, impairment, and biomarkers of integrity. Fluids Barriers CNS 17:69. doi: 10.1186/s12987-020-00230-3

Kim, K. A., Shin, D., Kim, J. H., Shin, Y. J., Rajanikant, G. K., Majid, A., et al. (2018). Role of autophagy in endothelial damage and blood-brain barrier disruption in ischemic stroke. Stroke 49, 1571-1579. doi: 10.1161/ STROKEAHA.117.017287

Kirk, S. J., Regan, M. C., and Barbul, A. (1990). Cloned murine T lymphocytes synthesize a molecule with the biological characteristics of nitric oxide. Biochem. Biophys. Res. Commun. 173, 660-665. doi: 10.1016/S0006-291X(05)80086-5

Kuthati, Y., Davuluri, V. N. G., Yang, C. P., Chang, H. C., Chang, C. P., and Wong, C. S. (2019). Melatonin MT2 receptor agonist IIK-7 produces antinociception by modulation of ROS and suppression of spinal microglial 
activation in neuropathic pain rats. J. Pain Res. 12, 2473-2485. doi: 10.2147/ JPR.S214671

Lakhan, S. E., Kirchgessner, A., and Hofer, M. (2009). Inflammatory mechanisms in ischemic stroke: therapeutic approaches. J. Transl. Med. 7:97. doi: 10.1186/1479-5876-7-97

Lapointe, J., Roy, M., St-Pierre, I., Kimmins, S., Gauvreau, D., MacLaren, L. A., et al. (2006). Hormonal and spatial regulation of nitric oxide synthases (NOS; neuronal NOS, inducible NOS, and endothelial NOS) in the oviducts. Endocrinology 147, 5600-5610. doi: 10.1210/en.2005-1548

Liu, J., Jin, X., Liu, K. J., and Liu, W. (2012). Matrix metalloproteinase-2mediated occludin degradation and caveolin-1-mediated claudin-5 redistribution contribute to blood-brain barrier damage in early ischemic stroke stage. J. Neurosci. 32, 3044-3057. doi: 10.1523/JNEUROSCI.6409-11.2012

Liu, J., Weaver, J., Jin, X., Zhang, Y., Xu, J., Liu, K. J., et al. (2016). Nitric oxide interacts with caveolin-1 to facilitate autophagy-lysosome-mediated claudin-5 degradation in oxygen-glucose deprivation-treated endothelial cells. Mol. Neurobiol. 53, 5935-5947. doi: 10.1007/s12035-015-9504-8

Lochhead, J. J., Yang, J., Ronaldson, P. T., and Davis, T. P. (2020). Structure, function, and regulation of the blood-brain barrier tight junction in central nervous system disorders. Front. Physiol. 11:914. doi: 10.3389/fphys.2020.00914

Lv, J., Hu, W., Yang, Z., Li, T., Jiang, S., Ma, Z., et al. (2018). Focusing on claudin-5: a promising candidate in the regulation of BBB to treat ischemic stroke. Prog. Neurobiol. 161, 79-96. doi: 10.1016/j.pneurobio.2017.12.001

Nitta, T., Hata, M., Gotoh, S., Seo, Y., Sasaki, H., Hashimoto, N., et al. (2003). Size-selective loosening of the blood-brain barrier in claudin-5-deficient mice. J. Cell Biol. 161, 653-660. doi: 10.1083/jcb.200302070

Nizet, V., and Johnson, R. S. (2009). Interdependence of hypoxic and innate immune responses. Nat. Rev. Immunol. 9, 609-617. doi: 10.1038/nri2607

Robinson, M. A., Baumgardner, J. E., and Otto, C. M. (2011). Oxygen-dependent regulation of nitric oxide production by inducible nitric oxide synthase. Free Radic. Biol. Med. 51, 1952-1965. doi: 10.1016/j.freeradbiomed.2011.08.034

Shi, Q., Cheng, Q., and Chen, C. (2021). The role of autophagy in the pathogenesis of ischemic stroke. Curr. Neuropharmacol. 19, 629-640. doi: 10.2174/157015 9X18666200729101913

Steinz, M. M., Santos-Alves, E., and Lanner, J. T. (2020). Skeletal muscle redox signaling in rheumatoid arthritis. Clin. Sci. 134, 2835-2850. doi: 10.1042/ CS20190728

Sweeney, M. D., Zhao, Z., Montagne, A., Nelson, A. R., and Zlokovic, B. V. (2019). Blood-brain barrier: from physiology to disease and back. Physiol. Rev. 99, 21-78. doi: 10.1152/physrev.00050.2017

Takeda, N., O’Dea, E. L., Doedens, A., Kim, J. W., Weidemann, A., Stockmann, C., et al. (2010). Differential activation and antagonistic function of HIF-\{alpha\} isoforms in macrophages are essential for NO homeostasis. Genes Dev. 24, 491-501. doi: 10.1101/gad.1881410

Traweger, A., Fang, D., Liu, Y. C., Stelzhammer, W., Krizbai, I. A., Fresser, F., et al. (2002). The tight junction-specific protein occludin is a functional target of the E3 ubiquitin-protein ligase itch. J. Biol. Chem. 277, 10201-10208. doi: 10.1074/jbc.M111384200

Wang, P., Shao, B. Z., Deng, Z., Chen, S., Yue, Z., and Miao, C. Y. (2018). Autophagy in ischemic stroke. Prog. Neurobiol. 163-164, 98-117. doi: 10.1016/j. pneurobio.2018.01.001
Wilhelm, I., Nyul-Toth, A., Suciu, M., Hermenean, A., and Krizbai, I. A. (2016). Heterogeneity of the blood-brain barrier. Tissue Barriers 4:e1143544. doi: $10.1080 / 21688370.2016 .1143544$

Xu, M. C., Gao, X. F., Ruan, C., Ge, Z. R., Lu, J. D., Zhang, J. J., et al. (2015). miR-103 regulates oxidative stress by targeting the BCL2/adenovirus E1B $19 \mathrm{kDa}$ interacting protein 3 in HUVECs. Oxid. Med. Cell. Longev. 2015:489647. doi: 10.1155/2015/489647

Yamamoto, Y., Konig, P., Henrich, M., Dedio, J., and Kummer, W. (2006). Hypoxia induces production of nitric oxide and reactive oxygen species in glomus cells of rat carotid body. Cell Tissue Res. 325, 3-11. doi: 10.1007/ s00441-006-0178-4

Yang, C., Hawkins, K. E., Dore, S., and Candelario-Jalil, E. (2019a). Neuroinflammatory mechanisms of blood-brain barrier damage in ischemic stroke. Am. J. Phys. Cell Physiol. 316, C135-C153. doi: 10.1152/ajpcell.00136.2018

Yang, Z., Huang, C., Wu, Y., Chen, B., Zhang, W., and Zhang, J. (2019b). Autophagy protects the blood-brain barrier through regulating the dynamic of claudin-5 in short-term starvation. Front. Physiol. 10:2. doi: 10.3389/fphys.2019.00002

Yang, Z., Lin, P., Chen, B., Zhang, X., Xiao, W., Wu, S., et al. (2020). Autophagy alleviates hypoxia-induced blood-brain barrier injury via regulation of CLDN5 (claudin 5). Autophagy 1-20. doi: 10.1080/15548627.2020.1851897 [Epub ahead of print]

Yang, Z., Wu, S., Fontana, F., Li, Y., Xiao, W., Gao, Z., et al. (2021). The tight junction protein claudin-5 limits endothelial cell motility. J. Cell Sci. 134:jcs248237. doi: $10.1242 /$ jcs. 248237

Yu, P., Deng, J., Cai, J., Zhang, Z., Zhang, J., Khan, M. H., et al. (2019). Anticancer and biological properties of a $\mathrm{Zn}$-2,6-diacetylpyridine bis(thiosemicarbazone) complex. Metallomics 11, 1372-1386. doi: 10.1039/C9MT00124G

Zhan, R., Zhao, M., Zhou, T., Chen, Y., Yu, W., Zhao, L., et al. (2018). Dapsone protects brain microvascular integrity from high-fat diet induced LDL oxidation. Cell Death Dis. 9:683. doi: 10.1038/s41419-018-0739-y

Zhang, J., Zhang, C., Jiang, X., Li, L., Zhang, D., Tang, D., et al. (2019). Involvement of autophagy in hypoxia-BNIP3 signaling to promote epidermal keratinocyte migration. Cell Death Dis. 10:234. doi: 10.1038/s41419-019-1473-9

Conflict of Interest: The authors declare that the research was conducted in the absence of any commercial or financial relationships that could be construed as a potential conflict of interest.

Publisher's Note: All claims expressed in this article are solely those of the authors and do not necessarily represent those of their affiliated organizations, or those of the publisher, the editors and the reviewers. Any product that may be evaluated in this article, or claim that may be made by its manufacturer, is not guaranteed or endorsed by the publisher.

Copyright (c) $2021 \mathrm{Yu}, \mathrm{Li}$, Zhong, Li, Chen and Zhang. This is an open-access article distributed under the terms of the Creative Commons Attribution License (CC BY). The use, distribution or reproduction in other forums is permitted, provided the original author(s) and the copyright owner(s) are credited and that the original publication in this journal is cited, in accordance with accepted academic practice. No use, distribution or reproduction is permitted which does not comply with these terms. 